\title{
Theory of mind in bilingual and monolingual preschool children
}

\begin{abstract}
This research examined whether theory of mind (ToM) development differs in bilingual and monolingual preschool children. Three false belief tasks were given to 163 Kurdish-Persian bilingual and Persian monolingual preschool children. Bilingual children performed significantly better than monolingual children in their ToM. Hierarchical multiple regression analysis revealed that, bilingualism contributed significantly to the prediction of preschoolers' ToM development when age and verbal ability were controlled.
\end{abstract}

Keyword: Theory of mind; Bilingualism; Preschooler; False belief; Verbal ability 